\title{
ARTIGOS
}

\section{ACHADOS NEUROPATOLÓGICOS NA SÍNDROME DA IMUNODEFICIÊNCIA ADQUIRIDA (SIDA): REVISÃO DE 138 CASOS}

\author{
Marco V. Wainstein, Lisiane Ferreira, Luciano Wolfenbuttel, Liane \\ Golbspan, Eduardo Sprinz, Matias Kronfeld e Maria Isabel Edelweiss
}

\begin{abstract}
Com o objetivo de determinar a incidência das direrentes afecções que podem atingir o Sistema Nervoso Central (SNC) na Síndrome da Imunodeficiência Adquirida (SIDA) realizouse um estudo retrospectivo através de necrópsias realizadas no Hospital de Clínicas de Porto Alegre. Para tanto, foram examinadas 138 necrópsias de pacientes portadores de SIDA que haviam morrido entre janeiro de 1985 e dezembo de 1990. Todos os cérebros foram analisados macroscópica e microscopicamente através da hematoxilina-eosina e, quando necessário, coloraçôes, especiais como Grocott, PAS, Giemsa e Ziehl-Nielsen, foram empregadas. As principais lesões encontradas foram: toxoplasmose cerebral em 29 casos (21\%); criptococose em 17 (12\%); tuberculose em dois (1\%) e um (0,7\%) de candidiase. Além destas lesões inflamatórias foram observados 15 casos (10\%) com lesões vasculares; oito (6\%) com gliose e sete (5\%) com achados sugestivos de encefalopatia pelo HIV. Podemos concluir que o SNC é um dos principais órgãos-alvo da SIDA e que a toxoplasmose cerebral é a principal forma de acometimento do SNC nestes pacientes.
\end{abstract}

Palavras-chaves: SIDA. Sistema nervoso central. Toxoplasmose cerebral.

As evidências atuais apontam para o SNC como um frequente órgão-alvo para as manifestações clínico-morfológicas da Síndrome da Imunodeficiência Adquirida (SIDA) ${ }^{7815}$. O envolvimento do SNC varia entre 60 a $90 \%$ em trabalhos realizados através de necrópsia ${ }^{111} 1214$. Os sintomas neurológicos podem ser, inclusive, a primeira manifestação da SIDA $^{3}$. Além das infecções oportunísticas como toxoplasmose, criptococose, candidíase, citomegalovirose e outras, o SNC é um importante sítio para a infecção pelo vírus da imunodeficiência adquirida $(\mathrm{HIV})^{245710121417 .}$

Dentre estas infecções oportunísticas, a toxoplasmose tem sido apontada como a principal causa de lesão cerebral associada à SIDA ${ }^{2}$. As análises neuropatológicas são, portanto, de importância fundamental não apenas para o estabelecimento de um diagnóstico acurado como também para elucidar a patogênese da lesão cerebral.

\footnotetext{
Trabalho realizado na Unidade de Doenças Infecciosas e Serviço de Anatomia Patológica do Hospital de Clínicas de Porto Alegre (HCPA).

Auxilio: FAPERGS e PROPESP

Endereço para correspondência: Dra. Maria Isabel Edelweiss. Serviço de Anatomia Patológica/HCPA. R. Ramiro Barcelos 2350 subsolo, 90035-003 Porto Alegre, RS, Brasil. Recebido para publicação em 17/01/92.
}

Este estudo tem como objetivo determinar a incidência das diversas afecções do SNC em pacientes portadores de SIDA através do diagnóstico por necrópsia.

\section{MATERIAIS E MÉTODOS}

Este é um estudo observacional retrospectivo onde foram analisadas as necrópsias de pacientes com SIDA realizadas no período de janeiro de 1985 a dezembro de 1990 no Serviço de Anatomia Patológica do Hospital de Clínicas de Porto Alegre (HCPA), correspondendo a 138 casos de um total de 256 necrópsias de SIDA já realizadas por este Serviço. Os únicos critérios de inclusão no estudo foram o diagnóstico de SIDA e a autorização para necrópsia. Foram feitos cortes seriados dos cérebros após fixação em formalina a $10 \%$ por um período médio de 30 dias. As lâminas foram rotineiramente coradas pela hematoxilina-eosina, e quando necessário, realizaram-se técnicas especiais de coloração tais como PAS, Grocott, Giemsa e Ziehl-Nielsen.

\section{RESULTADOS}

Das 138 necrópsias avaliadas, apenas nove pacientes eram do sexo feminino. Os principais 
Wainstein MV, Ferreira L, WolfenbuttelL, GolbspanL, Sprinz E, Kronfeld M, Edelweiss MI. Achados neuropatológicos na síndrome da imunodeficiência adquirida (SIDA): revisão de 138 casos. Revista da Sociedade Brasileira de Medicina Tropical 25:95-99, abr-jun, 1992.

achados neuropatológicos com suas respectivas incidências estão listados na Tabela 1. Em 81 casos (59\%) havia algum tipo de envolvimento do SNC. As infecções oportunísticas como um todo foram responsáveis pelo maior número de casos (35\%). Dentre estas, a mais prevalente foi a toxoplasmose cerebral, presente em 29 (21\%) casos. Neste grupo, criptococose foi diagnosticada em 17 , tuberculose em dois e candidíase em um paciente. A toxoplasmose cerebral foi morfologicamente estadiada (Tabela 2). O estágio 1 (5 casos) consistiu de abscesso necrosante com áreas de necrose mal delimitadas $\mathrm{e}$ com escasso infiltrado inflamatório e abundante número de taquizoítos. O estágio 2 (10 casos) representou o abscesso em organização com um halo de macrófagos e linfócitos circundando a área de necrose com um número variável de taquizoítos. No abscesso crônico ou terceiro estágio (14 casos) podem ser encontrados eventuais nódulos microgliais e raros toxoplasmas encistados (bradizoítos) e isolados (taquizoítos).

A lesão criptocóccica caracterizou-se pela formação de cistos intraparenquimatosos contendo numerosos fungos facilmente identificados pela $\mathrm{HE}$ ou pelo Grocott e escassa reação inflamatória. Estes cistos eram evidenciados macroscopicamente podendo atingir diferentes diâmetros. As meninges esboçavam um aspecto gelatinoso típico desta infecção.

$\mathrm{Na}$ meningite por cândida vêem-se pseudohifas do fungo com ausếncia de reação inflamatória. $\mathrm{Na}$ meningite tuberculosa foram observados numerosos macro ou micronódulos com necrose caseosa central e escasso infiltrado inflamatório mononuclear. Em nenhum deles foram identificadas células gigantes de Langhans. A pesquisa de BAAR

Tabela 1 - Principais afecções do SNC na SIDA - 138 casos/HCPA*.

Diagnóstico

Sem anormalidades

Infecções oportunísticas

toxoplasmose

criptococose

tuberculose

candidíase

Lesões vasculares

hemorragias

aterosclerose

trombose venosa

Gliose

Encefalopatia por HIV

Atrofia cortical

Neoplasias

Meningite bacteriana
$\mathrm{N}^{\circ}$ de casos

57

41,0

49

29

17

2

1

15

12

2

1

8

35,0

21,0

12,0

1,0

0,7

10,0

8,0

1,0

0,7

6,0

7

5,0

6

\footnotetext{
* Resultados obitidos através de necrópsias.
} 
Wainstein MV, Ferreira L, WolfenbuttelL, Golbspan L, Sprinz E, Kronfeld M, Edelweiss MI. Achados neuropatológicos na síndrome da imunodeficiência adquirida (SIDA): revisão de 138 casos. Revista da Sociedade Brasileira de Medicina Tropical 25:95-99, abr-jun, 1992.

Tabela 2 - Toxoplasmose cerebral - estadiamento do diagnóstico à necrópsia.

\begin{tabular}{lcc}
\hline Fase & $\mathrm{N}^{\circ}$ de casos & Percentagem (\%) \\
\hline I & 5 & 3,0 \\
II & 10 & 7,0 \\
III & 14 & 11,0 \\
\hline Total & 29 & 21,0 \\
\hline
\end{tabular}

revelou intensa positividade na coloração pelo Ziehl-Nielsen.

Além das lesões inflamatórias, as vasculares, como um todo, perfizeram um total de 15 casos (10\%), tendo sido encontrados 12 com hemorragias, dois com aterosclerose em vasos da base e um caso de trombose venosa. Foram feitos ainda os seguintes diagnósticos: oito casos (6\%) de gliose; sete $(5 \%)$ com achados sugestivos de encefalopatia pelo HIV; seis (4\%) com atrofia cortical; três $(2 \%)$ neoplasias, sendo dois linfomas e um ependimoma e, ainda, um $(0,7 \%)$ com meningite bacteriana onde não foi possivel identificar o agente.

Em nove pacientes foi encontrada mais de uma afecção no SNC (Tabela 3). Os diagnósticos concomitantes mais frequentes foram criptococose e toxoplasmose e toxoplasmose e hemorragias, ambos presentes em dois casos.

\section{DISCUSSÃO}

Aproximadamente $60 \%$ dasnecrópsias revisadas apresentaram algum tipo de envolvimento do SNC, demonstrando que este é um importante alvo na SIDA. Nossos resultados estão de acordo com outros estudos realizados anteriormente $\begin{array}{llll}1 & 11 & 12 & 14\end{array}$

Em termos individuais, a toxoplasmose cerebral foi a afecção do SNC mais frequente. Deve-se enfatizar, entretanto, que os resultados encontrados podem subestimar a nossa realidade, já que não foram empregadas técnicas especiais de imunohistoquímica, especialmente no que diz respeito ao citomegalovívus, a toxoplasmose e a encefalopatia pelo HIV, pois todas estas são

Tabela 3 - Casos com mais de um diagnóstico. Necrópsias em pacientes com SIDA.

Diagnóstico

$\mathrm{N}^{\circ}$ de casos

Criptococose + toxoplasmose

Toxoplasmose + hemorragias

Hemorragias + candidíase

Atrofia cortical + aterosclerose

Gliose + encefalopatia por HIV

Hemorragias + atrofia cortical

Criptococose + encefalopatia por HIV 
Wainstein MV, Ferreira L, WolfenbuttelL, Golbspan L, Sprinz E, Kronfeld M, Edelweiss MI. Achados neuropatológicos na sindrome da imunodeficiência adquirida (SWA): revisão de 138 casos. Revista da Sociedade Brasileira de Medicina Tropical 25:95-99, abr-jun, 1992.

condições cujo padrão-ouro de diagnóstico envolve técnicas de imunoperoxidase.

Os padrões morfológicos da toxoplasmose cerebral no nosso estudo são semelhantes aqueles encontrados e previamente descritos por Navia e cols $^{13}$. Conforme estes autores, estes padrões podem refletir a correlação existente entre a duração do tratamento antitoxoplasma e a gravidade da lesão. Desde modo, acredita-se que a lesão toxoplásmica no SNC represente a reativação de infecção latente e não adquirida após a infecção pelo HIV ${ }^{9} 18$.

A encefalopatia pelo HIV é representada pór duas manifestações neuropatológicas, descritas como encefalite multifocal de células gigantes ${ }^{3}$ é leucoencefalopatia difusa progressiva ${ }^{6}$. Em nosso estudo, foi diagnosticado um caso de encefalite multifocal, onde se detectaram focos de infiltrados de células gigantes multinucleadas em área subependimária, considerados por alguns autores como patognomônicos da encefalopatia pelo HIV ${ }^{16}$.

Os achados patológicos considerados sugestivos de leucoencefalopatia difusa progressiva encontrados em seis necrópsias foram: espongiose, gliose, nódulos microgliais e áreas de desmielinização. Os nódulos microgliais podem também estar associados à infecção pelo citomegalovírus e toxoplasma, não sendo exclusivos da encefalopatia pelo HIV ${ }^{7}$. O termo encefalopatia pelo HIV, segundo Lantos e cols $^{8}$ é preferido em relação às designações como complexo demencial da SIDA ou encefalite subaguda, desde que estes últimos apresentam conotações clínicas que nem sempre são substanciadas pelas evidências morfológicas.

Os estudos neuropatológicos podem apresentar importantes implicações na prática clínica da SIDA: As evidências substanciais de que o SNC possa representar um sítio principal de acometimento nos pacientes com SIDA, faz com que as avaliações neurológicas clínicas e radiológicas e especialmente, à necrópsia sejam fundamentais para a correta avaliação da doença bem como o prognóstico e diagnóstico acurados. Os estudos por necrópsia permitem não só o diagnóstico, que em muitas oportunidades não foi realizado em vida, como também a compreensão mais detalhada da patogênese da lesão, um conhecimento que pode ser fundamental para o tratamento e a prevenção da doença. Concluindo, nosso estudo através de necrópsia confirma dados de outros autores e procura destacar a necessidade do emprego de técnicas como tomografia computadorizada e/ou ressonância magnética com intuito do diagnóstico precoce das lesões do SNC. Estudos comparativos dos achados de necrópsia com os de tomografia poderão ainda ampliar a eficácia do diagnóstico de afecções do SNC na SIDA.

\section{SUMMARY}

This is an autopsys study performed in a retrospective fashion to determine the incidence of diseases that could affect the Central Nervous System in AIDS. For this purpose, 138 autopsies of patients with AIDS performed at the "Hospital de Clínicas de Porto Alegre", Brazil, between january $/ 85$ and december 190 were studied. All the brains were evaluated macroscopically and microscopically mainly through hematoxylin-eosin staining and if necessary special tecniques like PAS, Grocott, Giemsa and Ziehl-Nielsen were done. Results have revealed 29 (21\%) cases with cerebral toxoplasmosis; cryptococosis in 17 (12\%); tuberculosis in two (1\%) and one case $(0,7 \%)$ of candidiasis. Besides these inflammatory lesions, 15 (10\%) presented vascular lesion; $8(6 \%)$ had gliosis and $7(5 \%)$ cases had suggestive findings of HIV-encephalopath. We can conclude that the CNS is a important target affected by AIDS and that cerebral toxopalsmosis is the principal disease in the CNS in AIDS patientes.

Key-words: AIDS. Centralnervoussystem. Cerebral toxoplasmosis.

\section{REFERENCIAS BIBLIOGRAFICAS}

1. Anders KH, Guerra WF, Tomiyasu U, Verity MA, Vinters HV. The neuropathology of AIDS: UCLA experience and review. American Journal of Pathology 124:537-558, 1986.

2. Bishburg E, Robert HK, Slim J, Perez G, Johnson E. Brain lesions in patients with acquired immunodeficiency syndrome. Archives of Internal
Medicine 149:941\943, 1989.

3. Budka $H$, Constanzi G, Cristina S, Lechi A, Parravicini C, Trabattoni R, Vago L. Brain pathology induced by infection with the human immunodeficiency virus (HIV). A histological immunocytochemical, and electron microscopical study of 100 autopsy cases. Acta Neuropathologica 
Wainstein MV, Ferreira L, WolfenbuttelL, Golbspan L, Sprinz E, Kronfeld M, Edelweiss MI. Achados neuropatológicos na sindrome da imunodeficiência adquirida (SWDA): revisão de 138 casos. Revista da Sociedade Brasileira de Medicina Tropical 25:95-99, abr-jun, 1992.

75:185-198, 1987.

4. Gonzales MF, Davis RL. Neuropathology of acquired immunodeficiency syndrome. Neuropathology and Applied Neurobiology 14:345363, 1988.

5. Gray F, Ghererdi R, Scaravilli F. The neuropathology of acquired immunodeficiency syndrome (AIDS): a review. Brain 111:245-266, 1988.

6. Kleihues P, Lang W, Burger PC, Budka H, VogtM, Mourer R, Luthy R, Siegenthaler W. Progressive diffuse leukoencephalopathy in patients with acquired immunodeficiency syndrome (AIDS). Acta Neuropathologica 68:333-339, 1985.

7. Lang W, Miklossy J, Deruaz JP, Pizzolato GP, Probst A, Schaffner T, Gessaga E, Kleihues P. Neuropathologica 77:379-390, 1989.

8. Lantos PL, McLaughlin JE, Scholtz CL, Berry CL, Tighe JR. Neuropathology of the brain in HIV infection. Lancet 11:309-311, 1989.

9. Luft BJ, Brooks RG, Conley FK, McCabe RE, Remington JS. Toxoplasmic encephalitis in patients with acquired immunodeficiency syndrome. Journal of the American Medical Association 2532:913917, 1984.

10. Michaels J, Sharer LR, Epstein LG. Human immunodefiency virus type 1 (HIV-1) infection of the nervous system: a review. Immunodeficiency Review 1:71-104, 1988.

11. Monte SM, Ho DD, Schooley RT, Hirsh MS, Richardson EP. Subacute Encephalitis of AIDS and its relation to HTLV-III infection. Neurology 37:562-
$569,1987$.

12. Navia BA, Cho ES, Petito CK, Price RW, The AIDS dementia complex, II: neuropathology Annals of Neurology 19:525-535, 1986.

13. Navia BA, Petito CK, Gold JWM, Cho ES, Jordan $\mathrm{BD}$, Price RW.Cerebral toxoplasmosis complicating the acquired immunodeficiency syndrome: clinical and neuropathological findings of 27 patients. Annals of Neurology 19:224-238, 1986.

14. Petito CK, Cho ES, Lehmann W, Navia BA, Price RW. Neuropathology of acquired immunodefiency syndrome (AIDS): an aautopsy review. Journal of Neuropathology and Experimental Neurology 45:635-646, 1986.

15. Rosemberg S, Lopes MBS, Tsanaclis AM. Neuropathology of acquired immunodeficiency syncrome (AIDS). Analysis of 22 Brazilian cases. Journal of the Neurological Sciences 76:187-198, 1986.

16 Sharer LR, Cho ES, Epstein LG. Multinucleated giant cells and HTLV-III in AIDS encephalopathy. Human Pathology 16:760, 1985.

17. Wiley CA, Nelson JA. Role of immunodefiency virus and cytomegalovirus in AIDS encephalitis. American Journal of Pathology 133:73-81, 1988.

18. Wong B, Gold JWM, Brown AE, Lange M, Fried R, Grieco M, Milvan D, Giron J, Tapper ML, Lerner CW, Armstrong D. Central nervous system toxoplasmosis in homosexual men and parenteral drug abusers. Annals of Internal Medicine 100:36$42,1984$. 